\title{
Manchmal zu wenig, manchmal auch zu viel
}

Fragestellung: Wie häufig werden Diabetiker unzureichend bzw. zu intensiv antihypertensiv behandelt?

Hintergrund: Meistens wird beklagt und moniert, dass die Behandlung von kardiovaskulären Risikofaktoren wie Blutdruck, Blutfettwerte und Rauchen nicht konsequent genug erfolgt, die Zielwerte nicht erreicht werden und der Behandlungserfolg deshalb suboptimal bleibt. Andererseits ist natürlich auch eine $\mathrm{zu}$ aggressive Therapie möglich. Denn ein zu niedriger Blutdruck, vor allem diastolische Werte unter $70 \mathrm{mmHg}$, geht mit vermehrten kardiovaskulären Komplikationen einher und muss deshalb vermieden werden.

Patienten und Methodik: Die Analyse stützt sich auf 977282 Diabetiker (Alter 18 bis 75 Jahre), die zwischen 2009 und 2010 in US-amerikanischen Veterans Administration (VA) Zentren behandelt wurden. Als adäquat galt eine Hochdrucktherapie, wenn beim Arztbesuch die Werte $<140 / 90,<150 / 65$ oder bei mindesten 4 Antihypertensiva $<150 \mathrm{mmHg}$ lagen, wenn bei höheren Werten innerhalb von 90 Tagen die Therapie (Dosiserhöhung, Medikamentenwechsel) intensiviert wurde oder der Blutdruck bei Kontrolle $<140 / 90 \mathrm{mmHg}$ lag. Eine Übertherapie lag vor, wenn der Blut-

Kerr EA, Lucatorto MA, Hollrman R et al.

Monitoring Performance for Blood Pressure Management Among Patients With Diabetes Mellitus. Arch Intern Med 2012;172(12): 938-45. druck $<130 \mathrm{mmHg}$ systolisch und $<65 \mathrm{mmHg}$ diastolisch lag, wenn mit mehr als drei Antihypertensiva behandelt wurde oder falls eine neue Therapie oder Dosiserhöhung innerhalb von 90 Tagen eingeleitet wurde.

\section{-Kommentar von Prof. Dr. med. Heinrich Holzgreve}

\section{Der Erfolg hat zwei Gesichter}

Die Tatsache, dass 94\% aller Diabetiker adäquat , zusätzlich $8 \%$ zu intensiv, behandelt wurden, bedeutet eine dramatische Verbesserung in den letzten Jahrzehnten. Dies ist möglicherweise dem besonderem Engagement in den VA-Zentren geschuldet. Leider trägt der Erfolg aber zwei Gesichter: Wer ein ehrgeiziges Ziel anstrebt, schießt manchmal auch über das Ziel hinaus. So hatten die Zentren mit den höchsten Raten adäquater Therapie auch signifikant höhere Raten von Übertherapie. Die Leitlinien haben bislang immer nur Blutdruckzielwerte definiert, die erreicht werden sollen. In Zukunft wird man aber auch Grenzen nennen müssen, die nicht unterschritten werden dürfen, damit der Nutzen nicht durch potenziell gefährliche Folgen geschmälert wird. Ein risikoträchtiger, gut belegter,

leider jedoch häufig vernachlässigter Aspekt ist ein zu niedriger diastolischer Blutdruck.

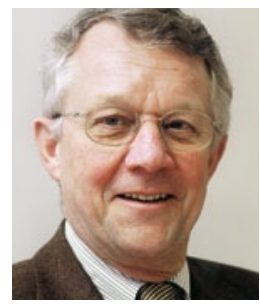

Prof. Dr. med. Heinrich Holzgreve

Internist, Kardiologische Praxis

Burgstr. 7, 80331 München

h.holzgreve@t-online.de 